\title{
CLASSIFICANDO CÉDULAS BRASILEIRAS (R\$) USANDO ANÁLISE DE IMAGEM POR SMARTPHONE
}

\author{
Bruno V. Vittorazzi ${ }^{a}$, Rayana A. Costa ${ }^{\mathrm{b}, \# \text {, Larissa M. Coelho }}{ }^{\mathrm{c}}$, Marsele M. Isidoro ${ }^{\mathrm{a}}$, Kássio M. G. Lima ${ }^{\mathrm{d}}$, Paulo R. Filgueiras ${ }^{\mathrm{b}}$ \\ e Wanderson Romão $0^{\mathrm{a}, \mathrm{b}, \mathrm{e}, *,(1)}$ \\ anstituto Federal do Espírito Santo, 29106-010 Vila Velha - ES, Brasil \\ 'Departamento de Química, Universidade Federal do Espírito Santo, 29075-910 Vitória - ES, Brasil \\ 'Instituto Federal do Espírito Santo, 29150-410 Cariacica - ES, Brasil \\ ${ }^{\mathrm{d} S}$ School of Pharmacy and Biomedical Sciences, University of Central Lancashire, Preston PR1 2HE, United Kingdom \\ 'Instituto Nacional de Ciência e Tecnologia Forense (INCT Forense), Brasil
}

Recebido em 10/12/2019; aceito em 21/01/2020; publicado na web em 03/04/2020

\begin{abstract}
CLASSIFYING BRAZILIAN BANKNOTES (R\$) USING SMARTPHONE IMAGE ANALYSIS. A new method to identify the authenticity of Brazilian banknotes $\left(\mathrm{R} \$\right.$ ) is proposed using digital image analysis by a smartphone application, Photometrix $^{\circledast}$, associated with chemometric tools. Authentic banknotes $(n=4)$ obtained in local bank and falsified $(n=12)$ by the author himself by means of three printers (laser-Deskjet ${ }^{\circledast}$, wax-Color Qube $^{\circledast}$ and inkjet-Deskjet ${ }^{\circledR}$ ) were used for the development of the methodology. An experimental planning was performed to evaluate the authenticity of the banknotes determining: the best region (holographic band, tactile region in the lower left and right upper number, effigy microimpressions, lower number left, animal figure, watermark and puzzle); Region of Interest (ROI) (16x16; $32 \times 32$ and 64x64 pixels), focal length (8, 12, 15 and $20 \mathrm{~cm})$ and variation of the smartphone (Motorola G5 Plus, Samsung S7 and LG K10). The best regions chosen were effigy microimpressions and round the animal. Promising results were obtained from ROI 16x16 pixels with focal length fixed at $10 \mathrm{~cm}$. Thus, the application presents promising features for analyzing the authenticity of banknotes by digital images making it fast, non-destructive, portable, with low cost, and can be accessed by all society.
\end{abstract}

Keywords: banknote; RGB image; PCA; smartphone.

\section{INTRODUÇÃO}

De acordo com o Código de Processo Penal brasileiro, documento é toda peça, seja de papel, plástico ou outro material, que contenha informações. ${ }^{1}$ A documentoscopia é uma vertente dentro da área da química forense que analisa, verifica e estuda a autenticidade de um documento. Essa difere-se de outras áreas de estudo de documentos por apresentar caráter nitidamente investigativo e criminal, procurando não só determinar a legitimidade do documento, como também os autores e os meios empregados. ${ }^{2}$

Segundo os criminologistas, com o passar do tempo, os crimes violentos, contra pessoa e propriedade, seriam substituídos por crimes de inteligência, como: a falsificação, o estelionato e a chantagem. Esses crimes possuem alta complexidade, devido a diversos contornos jurídicos que apresentam, bem como definir o conceito de documento, que possui uma ampla definição, não limitada a documentos de suporte em papel. Dentre os documentos que são passíveis de falsificação, as cédulas são as que provocam grande preocupação, pois afeta de forma considerável a economia de um país. ${ }^{3}$

A falsificação de papel-moeda tem causados impactos perceptíveis na sociedade, propiciando o desenvolvimento de outros crimes. Esse tipo de falsificação é considerado um dos maiores crimes financeiros e tem crescido mundialmente, tanto em quantidade quanto em qualidade. No Brasil, esse aumento foi notado a partir de 1994 com a introdução do Real (R\$) como moeda. ${ }^{4}$

Visando minimizar as falsificações, o Banco Central do Brasil, em 2010, apresentou medidas de segurança mais avançadas para o papel-moeda, por exemplo, elementos visíveis contra a luz branca, (Marca d’Água), o quebra-cabeça, fio de segurança, e elementos

*e-mail: wandersonromao@gmail.com

"e-mail alternativo: rayaalvarenga@gmail.com fluorescentes. O último pode ser facilmente identificado a partir do uso de lâmpada na região do ultravioleta. Na segunda geração de moedas do Real, essas medidas visam impor obstáculos mais sólidos as tentativas de falsificação. ${ }^{5}$ Porém, casos de falsificações de notas de R\$50 e R\$100 ainda são encontradas. ${ }^{6}$ Segundo dados do Banco Central, mais de 500 mil notas foram retidas de circulação em 2018 por apresentarem falsificações, sendo a região sudeste a que apresenta maior índice de falsificações seguida da região sul, nordeste e norte. ${ }^{7}$

As técnicas de espectroscopia molecular (infravermelho, Raman, fluorescência molecular) e de separação (cromatografia gasosa com detector de massas seletivo e eletroforese capilar com emissão de raios-X induzido por prótons) foram as mais empregadas em documentoscopia até os anos 2000. A partir de 2001, com o surgimento de fonte de ionização para espectroscopia de massas (MS - mass spectrometry), problemas complexos que existiam até o momento tornaram-se solucionáveis, como cruzamento de traços e datação de tintas. ${ }^{2}$ No Brasil, a polícia emprega técnicas baseadas nos espectros de reflectância na região do ultravioleta (UV) (200 a 390 nm), visível (400 a $800 \mathrm{~nm}$ ), e infravermelho (700 a $1000 \mathrm{~nm}$ ). Um equipamento comumente utilizado como ferramenta em investigações periciais é o Comparador Espectral de Vídeo (VSC), que permite a visualização de elementos de segurança, identificando adulterações. Além disso, o VSC pode ser usado como um simples espectrômetro, permitindo a aquisição de espectros de reflectância., ${ }^{3,8}$

Em documentoscopia, o grande desafio das técnicas analíticas é a necessidade de análises não destrutivas, rápidas, simples e de baixo custo. A preservação do material questionado é fundamental durante todo o processo de investigação. Assim, o desenvolvimento de metodologias analíticas com baixo ou nenhuma preparação de amostra, baixo custo de manutenção, fácil operação e a possibilidade de análise in situ é essencial na química forense. ${ }^{9}$ 
Com o avanço das tecnologias na área de visão computacional, tanto em software bem como em hardware, surge oportunidades de uso dessas tecnologias em diversas áreas. Dentre essas, a utilização de técnicas portáteis tem permitido melhorias significativas nos trabalhos de investigação. ${ }^{10}$ Os avanços recentes em tecnologias de aquisição e processamento de imagens, assim como a popularização de dispositivos móveis têm atraído à atenção da comunidade científica. $\mathrm{O}$ uso de imagens digitais vem sendo aplicado em análises que eram restritas a equipamentos como colorímetros, espectrofotômetros e fluorômetros. ${ }^{11}$

A análise de imagens digitais associada à análise multivariada permite o desenvolvimento de procedimentos simples, rápidos, não destrutivos e de baixo custo. $\mathrm{O}$ uso dessas imagens obtidas por scanners, câmeras digitais e outros, fornece resultados tão precisos quanto os instrumentos associados com análise multivariada. ${ }^{12}$

Os valores obtidos das análises podem ser entendidos como uma matriz determinada por linhas e colunas, em que os índices indicam um ponto na imagem, sendo denominados "elementos de imagem" ou pixels. Cada ponto ou pixels informa uma intensidade de cores no sistema, vermelho $(\mathrm{R})$, verde $(\mathrm{G})$ e azul (B), que quando associados propiciam diferentes cores. ${ }^{13-15}$ Assim, a relação entre imagens digitais no sistema de cores RGB e ensaios colorimétricos favorece a obtenção de dados qualitativos e medições analíticas quantitativas. Diversos estudos, utilizando o aplicativo PhotoMetrix como ferramenta de análises, vem sendo realizado como a caracterização de tanino por PCA demonstrando o desenvolvimento de um método rápido, não destrutivo e de baixo custo. ${ }^{12}$ Entre as aplicações reportadas estão: (i) quantificação de etanol em cachaça, ${ }^{16}$ (ii) quantificação de ferro em suplementos vitamínicos e aplicabilidade em cédulas de Real, podendo diferenciá-las de cédulas de Peso argentino de maneira eficaz por PCA, ${ }^{11}$ (iii) análise indireta de iodo no biodiesel, ${ }^{17}$ (iv) estabilidade térmica do leite, ${ }^{18}$ (v) identificação de taninos em amostras comerciais $^{12} \mathrm{e}$ (vi) monitoramento de flúor em água. ${ }^{19}$

Assim, visto a crescente incidência de casos de adulteração e fraudes em documentos, e a necessidade de métodos que exijam pouca manutenção, fácil operação, mínimo preparo de amostra, baixo custo, entre outros fatores, este trabalho objetiva avaliar uma nova metodologia para análise de autenticidade de cédulas de reais de $\mathrm{R} \$ 10,20,50$ e 100, buscando alcançar essas necessidades por meios de métodos quimiométricos aliados à análise de imagem digital.

\section{PARTE EXPERIMENTAL}

\section{Amostras}

Cédulas de $\mathrm{R} \$ 10,20,50$ e 100 foram selecionadas por apresentarem o maior índice de falsificações segundo dados do Banco Central do Brasil. Foram utilizadas 16 cédulas para cada valor, sendo 12 cédulas simuladas e quatro autênticas. As autênticas foram obtidas em banco local e as simulações foram reproduzidas de acordo com as imagens disponíveis na cartilha de treinamento da segunda família do real emitida pelo Banco Central do Brasil e produzidas em impressoras comerciais, do tipo Konica Minolta $654^{\circledR}\left(\right.$ LaserJet $\left.^{\circledR}\right)$, Xerox ColorQube $8880^{\circledR}$ (Cera) e uma Epson L656 ${ }^{\circledR}$ (Deskjet $^{\circledR}$ ) disponibilizadas pelo autor. Além das cédulas simuladas pelo autor, outras (R\$ 20, 50 e 100) compartilhadas pela Polícia Técnico-Científica do Estado do Espírito Santo, impressão offset, também foram disponibilizadas para o estudo, Tabela 1.

\section{Análise dos dados}

Para a análise dos dados utilizou-se o aplicativo PhotoMetrix ${ }^{\circledR}$ desenvolvido pelo grupo de pesquisa em quimiometria da Universidade
Tabela 1. Quantitativo de cédulas para o estudo de classificação de cédulas de real

\begin{tabular}{cccc}
\hline Cédula $(\mathrm{R} \$)$ & Autênticas & Simulações & Falsificadas \\
\hline 10 & 4 & 12 & 0 \\
20 & 4 & 12 & 2 \\
50 & 4 & 12 & 1 \\
100 & 4 & 12 & 8 \\
\hline Total & 16 & 48 & 11 \\
\hline
\end{tabular}

de Santa Cruz do Sul (RS, Brasil), disponível gratuitamente na loja de aplicativos Android e IOS (http://www.photometrix.com.br). O aplicativo contém ferramentas de análise multivariadas e permite realizar análise de componentes principais (PCA), regressão por mínimos quadrados parciais (PLS) e análise hierárquica de agrupamentos (HCA).

A interface do aplicativo inclui análises univariadas e multivariadas (Figura 1Sa). Na interface de configuração (Figura 1Sb), o usuário pode determinar a região de interesse, configurações da câmera, cadastrar um e-mail para exportar os dados, entre outros.

O método de análise por PCA realizado pelo aplicativo tem como principal característica rapidez e facilidade, contando com poucos passos até os dados finais, sem a necessidade de tratamento de imagens para interpretação dos resultados. As etapas envolvidas na análise são mostradas na Figura 2S. Para construção dos gráficos, ficou estabelecido a utilização dos canais R, G, B, H, S, V, L, I com dados centrados na média.

\section{Ensaios de otimização}

A otimização das análises foi realizada, com base na cédula de $\mathrm{R} \$ 50$, determinando a melhor região para aquisição da imagem (faixa holográfica, região tátil no número inferior esquerdo e número superior direito, microimpressões na efígie, número inferior esquerdo, figura do animal, marca d'água e quebra-cabeça), configuração da região de interesse (do inglês Region Of Interest - ROI) (16x16; 32x32; 64x64), distância focal $(8,12,15$ e 20 cm) e controle de luminosidade (laboratório com ambiente de luminosidade controlada e em campo). Após a otimização, realizou-se a análise nas cédulas dos outros valores (100, 20 e 10 reais) considerando os critérios que apresentaram melhores resultados anteriormente. Por fim, variou-se o modelo do smartphone para observar a influência deste nos resultados.

\section{Ensaio 1 - Determinação da melhor região da cédula}

A cédula de real apresenta regiões com medidas de segurança gráficas facilitando a identificação de possíveis adulterações, sendo que alguns pontos são compartilhados por todos os valores de cédulas. Dentre os diversos pontos alguns foram selecionados, na região do reverso e anverso, para o estudo proposto e os mesmos são mostrados na Figura 1.

A análise de melhor região para aquisição dos dados foi realizada com um smartphone Motorola G5 Plus, com câmera de 12 megapixels e sistema operacional Android 8.0. Para o estudo não há necessidade do preparo de amostra e as análises foram realizadas em ambiente controlado, sendo avaliado o efeito do controle de luminosidade com uma luminária LED de $3.5 \mathrm{~W}$.

\section{Ensaio 2 - Região de interesse (ROI) e distância focal}

Duas variáveis que foram observadas: o tamanho do spot, que pode variar entre $16 \times 16,32 \times 32$ e $64 \times 64$; e a distância focal entre a 


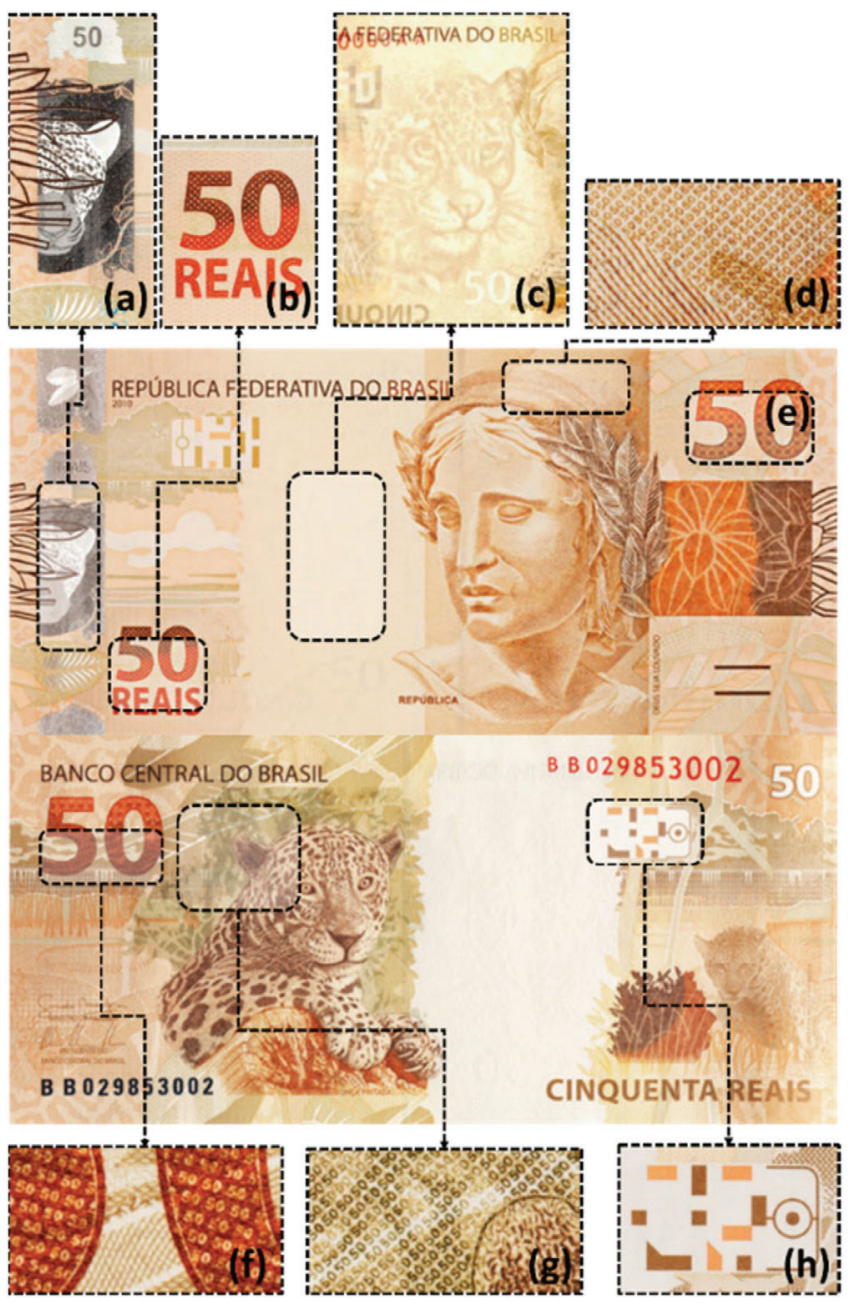

Figura 1. Regiões da cédula da melhor ROI para o estudo: (a) faixa holográfica, (b) região tátil - número inferior esquerdo, (c) marca d'água, (d) microimpressão - região da efígie, (e) região tátil - número superior direito, (f) microimpressão - número superior esquerdo, $(\mathrm{g})$ microimpressão - região da figura do animal, e $(h)$ quebra cabeça $(h)$

amostra e a câmera do smartphone utilizado. Portanto, a partir da análise na melhor região, foi proposto a análise para spots de 16x16, $32 \times 32$ e 64x64 pixels, assim como variação da distância entre $8 \mathrm{~cm}$ e $20 \mathrm{~cm}$, que compreende a distância mínima e máxima em que se verificou a diminuição do foco sobre a amostra.

\section{Ensaio 3 - Variação do modelo do smartphone}

Esse ensaio visa principalmente analisar a influência do smartphone utilizado na análise. Como as características da câmera dos aparelhos possuem diferentes opções, a reprodutibilidade dos resultados entre equipamentos diferentes deve ser considerada como uma das variáveis nas análises.

Para realizar esse ensaio, foram utilizados três smartphones de marcas diferentes, Motorola G5 Plus (câmera de 12 megapixels, abertura F 1.7, estabilização digital, resolução 4032x3024 pixel e sistema operacional Android 8.1 Oreo), um Samsung Galaxy S7 (câmera de 12 megapixels, abertura F 1.7, estabilização óptica, resolução 4290x2800 pixel e sistema operacional Android 8.0 Samsung Experience 9.0 Oreo), e um LG K10 (câmera de 13 megapixels, abertura F2.2, estabilização digital, resolução 4160x3120 pixel e sistema operacional Android 6.0). Nessa análise, foram utilizados os critérios encontrados nos ensaios 1 e 2 , assim, as configurações de ROI e distância focal foram $16 \times 16$ pixels e $10 \mathrm{~cm}$, respectivamente.

\section{Análise das cédulas de $\mathbf{R} \$ 10,20$ e 100}

A partir da escolha da melhor região de interesse da cédula de $\mathrm{R} \$ 50$, bem como análise da influência do tamanho do spot e distância focal entre amostra e smartphone, foram analisadas cédulas de $\mathrm{R} \$ 100$, 20 e 10. A metodologia utilizada foi similar ao descrito anteriormente. Um total de dezesseis cédulas para cada valor foi utilizado, sendo quatro amostras autênticas e 12 falsificadas (quatro para cada marca de impressora).

\section{RESULTADOS E DISCUSSÃO}

\section{Ensaio 1 - Determinação da melhor região da cédula}

Os resultados para determinação da melhor região de análise na cédula de $\mathrm{R} \$ 50$ revelaram que algumas regiões são mais consistentes e confiáveis quando procuramos discriminar as cédulas entre autênticas e falsificadas em grupos bem determinados (Figura 2, 3S e 4S). Os resultados foram obtidos a partir dos valores dos canais $\mathrm{R}, \mathrm{G}, \mathrm{B}, \mathrm{H}$, $\mathrm{S}, \mathrm{V}, \mathrm{L}$ e I com dados centrados na média. A Figura 2 mostra o gráfico de scores (PC1 vs. PC2) para as regiões do anverso e reverso. No anverso, temos a região de microimpressão na efígie (Figura 2a), com grupamentos separados e bem definidos, tanto para cédulas autênticas quanto falsificadas. A região da $\mathrm{PC} 2<0$ foi responsável por separar as cédulas autênticas enquanto toda a região da $\mathrm{PC} 1$ foi responsável por separar os três grupos de cédulas falsificadas. Pela análise do gráfico de loadings dos canais do anverso para região da PC1 (Figura 5Sa), pode-se observar que o canal $\mathrm{H}$ é o responsável pela separação das cédulas autênticas, enquanto os canais $\mathrm{R}, \mathrm{G}, \mathrm{B}$ determinam as cédulas falsificas. No reverso, temos a região de microimpressão entorno do animal (Figura 2b) com a mesma característica, sendo a $\mathrm{PC} 1>0$ responsável por separar as cédulas autênticas das falsificadas e toda a região da PC2 responsável por separar os três grupos de cédulas falsificadas. Analisando o gráfico de loadings para $\mathrm{PC} 1$ na região reverso (Figura 3Sb), temos os canais $\mathrm{H}$ e B como responsáveis pela identificação das cédulas autênticas, e os canais $\mathrm{G}$ e $\mathrm{R}$ como responsáveis pela identificação das cédulas falsificadas.

Os resultados obtidos corroboram com o estudo de Helfer e colaboradores, ${ }^{18}$ que conseguiram distinguir diferentes tipos de cédulas, em que as três primeiras PCs somaram 98,5\% da variância explicativa entre cédulas de Real e 98,75\% entre cédulas de Real e Peso argentino. Neste estudo, as duas primeiras PCs somaram 95,70\% da variância entre cédulas autênticas e falsificadas de $\mathrm{R} \$ 50$ quando analisado o anverso na região da microimpressão da efígie e $96,15 \%$ de variância considerando a região de microimpressão em torno do animal no reverso. Os valores em porcentagem das variâncias são menores quando comparados ao de Helfer e colaboradores. ${ }^{18}$ Porém, é importante destacar que a comparação foi realizada entre cédulas autênticas e falsificadas de cédulas do mesmo valor, o que torna o modelo mais complexo, justificando assim a menor variância dos valores encontrados.

\section{Ensaio 2 - Região de interesse e distância focal}

Uma das configurações para as análises é a ROI, que delimita o tamanho do local na imagem, no qual o aplicativo obtém os dados. Esse valor pode ser de $8 \times 8,16 \times 16,32 \times 32$ e $64 \times 64$ pixels. Para o ensaio, os valores de $16 \times 16,32 \times 32$ e $64 \times 64$ pixels foram escolhidos por apresentarem tamanho considerável para aquisição da imagem que contém a região dos elementos de segurança.

O gráfico de scores da PC1 vs. PC2 (com variância explicativa variando de $93,77 \%$ até $98,28 \%$ ), Figura 3 , mostram que os agrupamentos de amostras autênticas e falsas apresentam-se bem agrupados, 


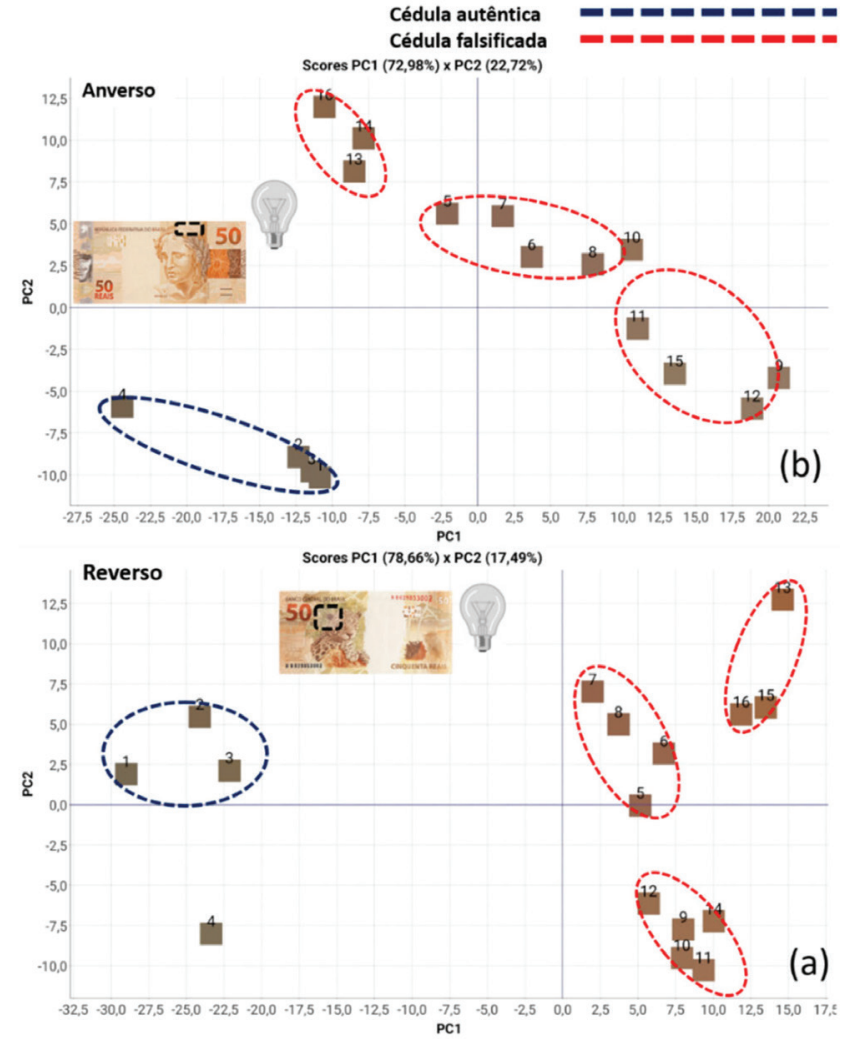

Figura 2. PCA da cédula de $R \$ 50$ contendo o melhor agrupamento das amostras: região (a) anverso de microimpressão na efígie; $e$ (b) reverso de microimpressão no entorno do animal

porém, pode-se destacar que a ROI de 16x16 pixels (Figura 3a,d) apresenta uma melhor distinção das cédulas, principalmente entre as amostras falsificadas. O mesmo não aconteceu para as amostras analisadas na região anverso ROI 32x32 e 64x64 pixels, Figuras 3e e 3 f, respectivamente.

Como a distância entre a aquisição da imagem e a câmera do smartphone pode ser uma variável importante que pode influenciar a qualidade final do resultado, ela também foi estudada. ${ }^{18}$ Aqui, determinou-se uma faixa de trabalho que compreende a distância máxima e mínima que é possível focar a amostra sem perda de resolução (valores de 8, 12, 15, e $20 \mathrm{~cm}$ ), Figura 4. Em geral, os resultados obtidos mostram uma excelente separação entre as cédulas de $\mathrm{R} \$ 50$ autênticas e falsas em todos os casos, provando que a variação da distância não influencia a qualidade final do resultado. Os valores da soma da variância explicada da PC1 e PC2 variaram de 89\% a 97\%.

\section{Ensaio 3 - Variação da marca do smartphone}

O estudo da influência do uso de diferentes smartphones também foi avaliado neste trabalho, sendo que a captura da imagem pode ser influenciada pela qualidade dos componentes eletrônicos da câmera. Pode-se observar na Figura 5, de uma maneira geral, que é possível determinar a autenticidade da cédula de $\mathrm{R} \$ 50$ independente da marca dos três smartphones analisados. No caso do smartphone da marca LG K10, o gráfico de PCA (Figura 5e-f) apresentou uma limitação para agrupar as cédulas autênticas e falsificadas. Mesmo apresentando maior quantidade de pixel, essa limitação pode estar associada ao conjunto de abertura da câmera e estabilização, que vão influenciar, respectivamente, na quantidade de luz que a lente permite entrar e na correção de movimentos na captura da imagem, sendo assim fatores determinantes na qualidade da imagem obtida e, consequentemente, na diferenciação das cédulas. Analisando os gráficos de scores da PC1 $x \mathrm{PC} 2$, é possível visualizar que ambas as PCs são importantes para determinar a autenticidade da cédula de $\mathrm{R} \$ 50$. Por outro lado, a região da PC1 é responsável pela diferenciação do tipo de falsificação empregada (impressoras laser, cera ou jato de tinta) tanto na região do anverso quanto no reverso. Para o smartphone da marca Samsung Galaxy S7 (Figura 5c-d) e Motorola G5 Plus (Figura 5a-b), grupos bem definidos também são observados. Por meio da análise dos scores da região da PC1xPC2, é possível constatar que PC2 é a componente determinante para separação das cédulas autênticas tanto no anverso quanto no reverso. Resultados semelhantes referente ao smartphone Samsung Galaxy S7 e Motorola G5 Plus estão relacionados à quantidade de pixel e pela abertura da câmera, configurações que são semelhantes entre os smartphones. É importante destacar que para uma imagem de resolução considerável, aspectos como abertura da câmera, estabilização, resolução, tamanho do sensor, detector, grande alcance dinâmico - HDR, dentre outros, devem ser considerados além da quantidade de pixels.

\section{Análise das cédulas de R\$10, 20 e 100}

A partir dos dados obtidos, foram observados que a região de microimpressão na efígie (anverso) e no entorno do animal (reverso) apresentaram melhor agrupamentos entre as cédulas autênticas e falsificadas, sendo essas as regiões utilizadas para o estudo das demais cédulas. Em relação aos dados da ROI, foi utilizado a condição ótima de 16x16 pixels. A variação da distância não foi determinante para alterações durante as análises, porém ficou determinada uma distância fixa de $10 \mathrm{~cm}$ entre a câmera do smartphone e a cédula. A partir desses critérios determinados pelos ensaios de otimização, foi possível construir um perfil de análise padrão, que pode ser utilizado para análises em série, facilitando o planejamento experimental.

Foi observado que o smartphone Motorola G5 Plus apresentou resultados mais consistentes na diferenciação dos grupos de cédulas de diferentes valores, como pode ser observado na Figura 6S.

A cédula de $R \$ 100$ (Figura 6Sa-b) apresenta boa diferenciação entre cédulas autênticas e falsificadas, em que a região da PC1 é a componente determinante na separação das imagens nas notas na região anverso e reverso, sendo influenciada pelos canais $R$ e G (Figura $7 \mathrm{~S}$ ), tendo uma variância de 97,40 até $99,15 \%$. Para a cédula de $\mathrm{R} \$ 20$ (Figura 6Sc-d), os grupos apresentam-se definidos, sendo separados principalmente pela região da PC2 (autênticas $v s$. falsificadas), sendo que os canais que influenciam na separação das cédulas autênticas são $\mathrm{G}$ e $\mathrm{H}$ no anverso e G, B e H no reverso somando uma variância explicativa de 90,27\% (Figura $8 \mathrm{Sa}$ ) e 92,3\% (Figura $8 \mathrm{Sb}$ ), respectivamente. Nas cédulas de $\mathrm{R} \$ 10$ (Figura 6Se-f), têm-se um agrupamento bem definido, sendo a região da PC2 a componente determinante para separação no anverso (autênticas $v s$. falsificadas) e a região da $\mathrm{PC} 1$ sendo determinante para o reverso. Os canais que influenciam a região da PC2 no anverso (Figura 9Sa) são G, B e H, enquanto temos o canal $\mathrm{H}$ (Figura 9Sb) influenciando a região da PC1 no reverso, apresentando uma variância explicativa somando as duas PC's de 94,33 até $95,62 \%$.

As cédulas de $\mathrm{R} \$ 10,20$ e 100 foram também analisadas nos smartphones Samsung Galaxy S7 e LG K10, e podemos visualizar os resultados dos scores PC1 vs. PC2 na Figura 10S e Figura 11S. Os resultados obtidos com o smartphone Samsung Galaxy S7 apresentam agrupamentos entre as cédulas, podendo classificá-las como autênticas e falsificadas para todas as cédulas investigadas. Porém, os resultados não apresentaram um agrupamento dos dados bem definidos como mostrado anteriormente utilizando o smartphone Motorola G5 Plus, Figuras 3-4. Já o smartphone LG K10 apresenta certa diferenciação entre grupos, porém não bem delimitados 

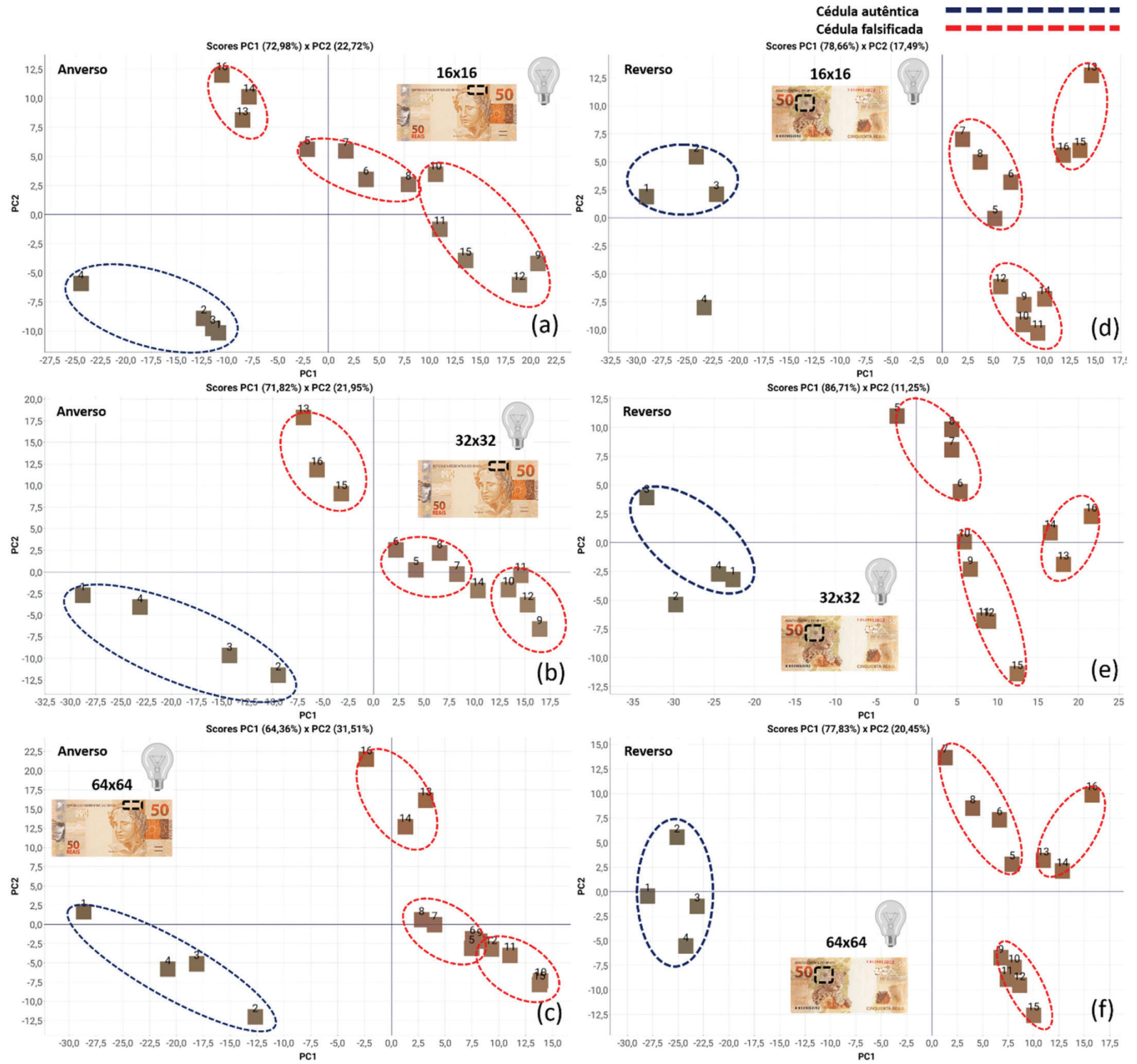

Figura 3. Variação do tamanho do spot na região de microimpressão da efígie de cédulas de R\$50 com ROI-16x16 pixels (a), $32 \times 32$ pixels (b), e $64 x 64$ pixels (c); e na região da microimpressão do entorno do animal com ROI - $16 x 16$ pixels (d), $32 x 32$ pixels (e), e $64 x 64$ pixels ( $f$ )

comprometendo a identificação entre autênticas e falsificadas de algumas cédulas como as de $\mathrm{R} \$ 20$ e 10 .

Tendo em vista que a falsificação de papel moeda é um grande problema de segurança pública de nível nacional, inseriu-se no estudo cédulas apreendidas pela Polícia Técnico-Científica do Estado do Espírito Santo. O objetivo é avaliar se o aplicativo é robusto frente a essas sofisticações no processo de fraudes de cédulas de real. As mesmas condições de análise foram consideradas e o smartphone para captura das imagens foi o Samsung S7.

A cédula de $R \$ 100$ (Figura 6a-b) apresenta certa separação entre cédulas autênticas e falsificadas, sendo que a região da PC2 é a componente determinante na separação na região anverso, sendo influenciada pelo canal R (Figura 12Sa), tendo uma variância de $97,2 \%$ de explicação. Na região do reverso o agrupamento das cédulas autênticas frente às falsificadas, deu-se pela região da PC1, sendo influenciada pelos canais $\mathrm{R}, \mathrm{Ge}$ B somando uma variância explicativa de $98,64 \%$ (Figura 12Sb). Observa-se por meio dos resultados obtidos que para a cédula de $\mathrm{R} \$ 100$ a região do reverso apresenta melhores agrupamentos, mesmo com uma cédula falsificada (impressão do tipo offset) inserida no grupo das autênticas, sendo assim passível de inferir que essa região é a mais adequada para classificação entre cédulas autênticas e falsificadas de $\mathrm{R} \$ 100$. Para cédula de $\mathrm{R} \$ 50$ (Figura 6c-d), os grupos (autênticas vs. falsificadas) apresentam-se definidos, sendo separados principalmente pela região da $\mathrm{PC} 1$, em que os canais que influenciam na separação das cédulas autênticas são $\mathrm{H}$ no anverso e $\mathrm{G}, \mathrm{B}$ e $\mathrm{H}$ no reverso somando uma variância de 96,15\% (Figura 13Sa) e 89,75\% respectivamente. Para a cédula de $\mathrm{R} \$ 50$, observa-se que a cédula compartilhada pela PCES (17) se apresenta bem separada das autênticas, visto que a mistura de cores da região selecionada (bege) é mais complexa para reprodução, o que dificulta a aproximação das cédulas falsificadas com relação as autênticas. Já para as cédulas de R \$20 (Figura 6e-f), observa-se que a melhor região para o verificar a diferenciação entre cédulas autênticas e falsificadas é o reverso, em que a região da PC1 é responsável em 

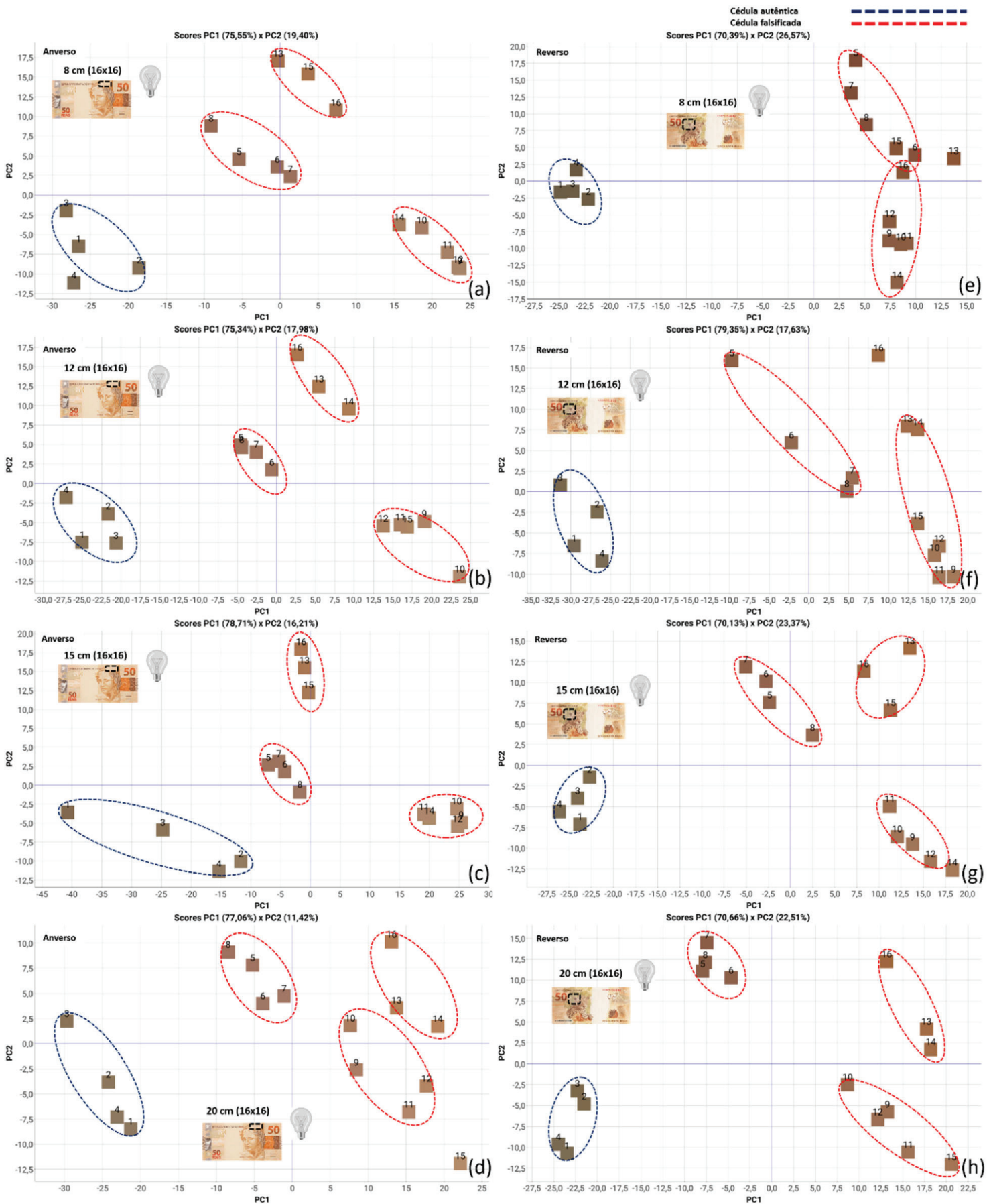

Figura 4. Estudo da distância entre a aquisição da imagem e a câmera do smartphone (Motorola G5 Plus) na cédula de R $\$ 50$ para a região da microimpressão efígie ( $8 \mathrm{~cm}(\mathrm{a}), 12 \mathrm{~cm}(\mathrm{~b}), 15 \mathrm{~cm}(\mathrm{c})$, e $20 \mathrm{~cm}(\mathrm{~d}))$ e na região da microimpressão do entorno do animal $(8 \mathrm{~cm}(\mathrm{e}), 12 \mathrm{~cm}(\mathrm{f}), 15 \mathrm{~cm}(\mathrm{~g}), e 20 \mathrm{~cm}(\mathrm{~h}))$

separar essas cédulas sendo influenciada pelos canais R, G, B e H (Figura 14S) totalizando uma variância de 72,5\% entre PC1 e PC3. Cabe ressaltar que algumas cédulas não apresentam grupamento dentro da classificação pelo tipo de impressão. Esse fato pode estar relacionado ao estado de conservação das cédulas, uma vez que com o passar do tempo e manuseio as mesmas apresentam desgastes e com isso a perda na intensidade da coloração.

Outro parâmetro avaliado foi com relação à análise em campo, uma vez que para a otimização realizou-se as análises em ambiente de luminosidade controlado. Observa-se que é possível identificar a separação entre cédulas autênticas e falsificadas mesmo em ambiente sem controle de luminosidade, ou seja, em análise em campo (Figura 15S). Essas análises foram realizadas apenas no anverso das cédulas de $\mathrm{R} \$ 20,50$ e 100 na região do número na parte inferior da cédula. Para cédulas de $\mathrm{R} \$ 20,50$ e 100 verifica-se que a região responsável pela separação é $\mathrm{PC} 1, \mathrm{PC} 2$ e PC1, respectivamente. Portanto, é possível verificar a grande potencialidade do aplicativo PhotoMetrix ${ }^{\circledR}$ para análise de falsificação de cédulas.

\section{CONCLUSÃO}

Nesse estudo foram utilizados um total de 75 amostras de cédulas de Real, sendo quatro amostras autênticas, 12 amostras simuladas e 11 amostras falsificadas para cada valor, todas analisadas a partir do aplicativo PhotoMetrix ${ }^{\circledR}$. Foi proposto um planejamento experimental no qual se variou a melhor região para aquisição da imagem (faixa 

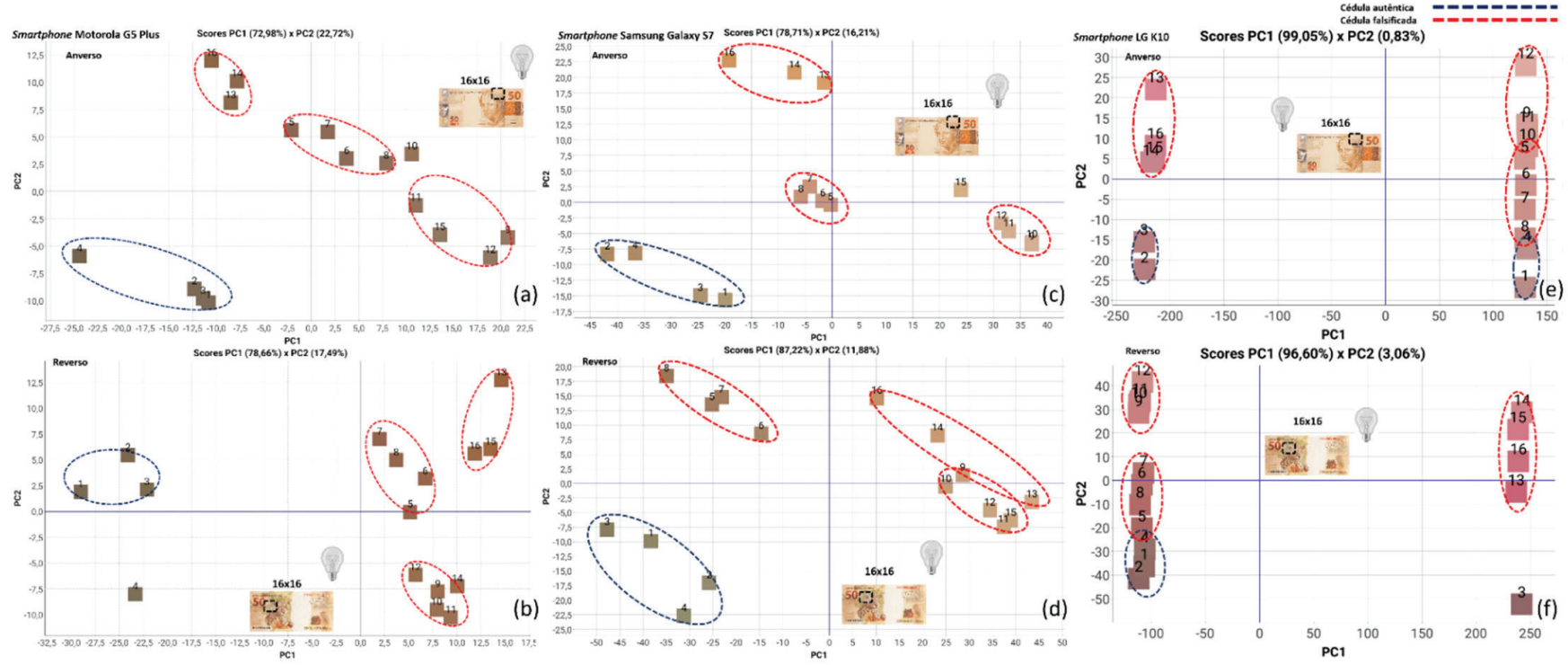

Figura 5. Variação da marca do smartphone. Motorola G5 Plus - região de microimpressão na efígie (a), Motorola G5 Plus - região de microimpressão no entorno do animal (b), Samsung Galaxy S7 - região de microimpressão da efígie (c), Samsung Galaxy S7, região de microimpressão animal (d), LG K10, região de microimpressão da efígie (e), LG K10, região de microimpressão no entorno do animal (f)
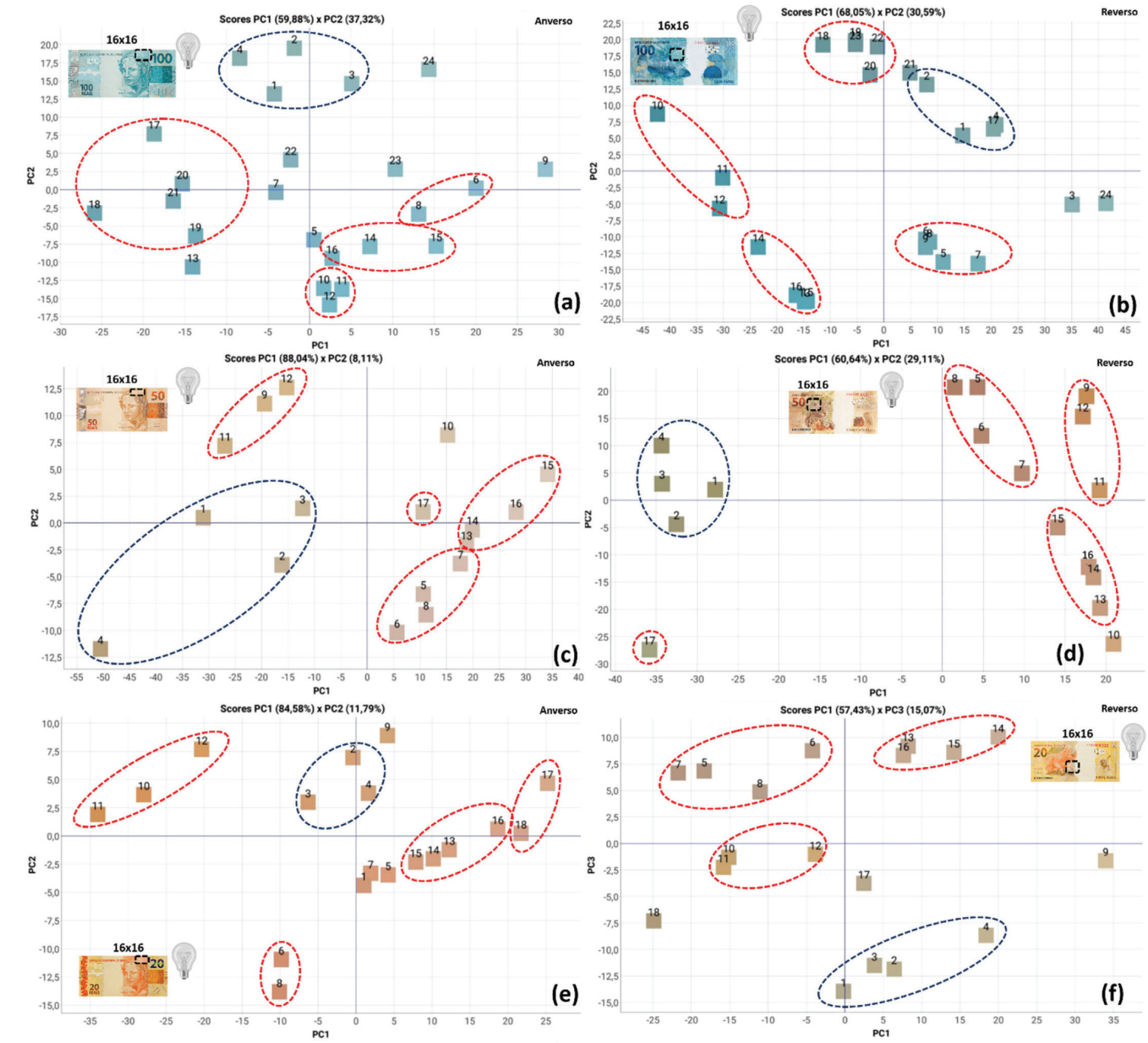

Figura 6. Análise de cédulas de $R \$ 100,50$ e 20 apreendidas, usando um smartphone Samsung Galaxy S7, ROI-16x16 pixels (distância de $10 \mathrm{~cm}$ da amostra): microimpressões na região da efígie $(a, c, e)$, e do entorno do animal $(b, d, f)$. Círculos azuis e vermelhos representam as notas autênticas e falsificadas, respectivamente 
holográfica, região tátil no número inferior esquerdo e número superior direito, microimpressões na efígie, número inferior esquerdo, figura do animal, marca d'água e quebra-cabeça), configuração da ROI (16x16; 32x32; 64x64), distância focal (8, 12, 15 e $20 \mathrm{~cm})$, modelo do smartphone e controle de luminosidade (laboratório com ambiente de luminosidade controlada). Cabe ressaltar que, após o estudo, determinou-se como melhor região para aquisição da imagem no anverso a microimpressão na efígie e no reverso microimpressão em torno do animal, configuração de $16 \times 16$ pixels, distância focal de $10 \mathrm{~cm}$ e luminosidade em ambiente controlado, para cédula de $\mathrm{R} \$ 50$ obtidas por simulações a cédulas autênticas. A variação do modelo do smartphone apresentou resultados pouco diferentes, mas o da marca Motorola G5 Plus destacou-se frente ao Samsung S7 e LG K10. Após a otimização do método, aplicou-se a utilização em amostras falsificadas apreendidas $(n=11)$ compartilhadas pela PCES. Assim, pode-se inferir que dentro do espaço amostral obtido pelo procedimento há classificação entre cédulas autênticas e falsificadas. O método proposto é simples, de fácil execução e baixo custo. Observaram-se resultados de excelente qualidade demonstrando o potencial do aplicativo PhotoMetrix ${ }^{\circledR}$ para a classificação de cédulas via análise colorimétrica de imagem digital associada a análise multivariada por PCA, uma vez que foi possível classificar cédulas autênticas e falsificadas utilizando a tecnologia disponível a favor da ciência e sociedade. Além disso, o aplicativo apresenta uma interface interativa proporcionando o usuário rápido aprendizado para realização das análises e obtenção dos resultados. Por fim, a metodologia proposta é não destrutiva, é rápida e de baixo custo, sendo uma alternativa viável para análises de autenticidade de cédulas in loco, visto que com os resultados obtidos em campo foi possível demonstrar que a luminosidade não é um fator determinante os dados obtidos, proporcionando maior praticidade e rapidez na obtenção dos resultados.

\section{MATERIAL SUPLEMENTAR}

Algumas imagens dos sistemas utilizados neste trabalho estão disponíveis, para melhor compreensão, em http://quimicanova.sbq. org.br, na forma de arquivo PDF, com acesso livre.

\section{AGRADECIMENTOS}

Os autores agradecem à CAPES (23038.007083/2014-40), à FAPES (EDITAL CNPq/FAPES N ${ }^{\circ}$ 23/2018 - PROGRAMA DE APOIO A NÚCLEOS EMERGENTES - PRONEM), e ao CNPq (422555/2018-5, e 305359/2017-7) pelo suporte financeiro. Os autores também gostariam de agradecer ao Núcleo de Competências em Química do Petróleo e ao LabPetro pelo uso de suas instalações e à Perita Criminal Jandira Maria de Oliveira Bone Brandão pelo fornecimento das cédulas apreendidas pela Polícia Civil do Estado do Espírito Santo.

\section{REFERÊNCIAS}

1. Fundamentos de Química Forense: Uma análise prática da química que soluciona crimes; Velho, J. A., Bruni, A. T., de Andrade, J. F., orgs.; $1^{\text {st }}$ ed., Millenium: Campinas, 2012.

2. Romão, W.; Schwab, N. V.; Bueno, M. I. M. S.; Sparrapan, R.; Eberlin, M. N.; Martiny, A.; Sabino, B. D.; Maldaner, A. O.; Quim. Nova 2011, 34, 1717.

3. Brandão, J. M. de O. B.; Almeida, N. S. M.; Dixini, P. V. M.; Baier, C. H. A.; Dias, H. P.; Bassane, J. F. P.; França, H. S.; Silva, S. R. C.; Aquije, G. M. F. V.; Romão, W.; Anal. Methods 2016, 8, 771.

4. Eberlin, L. S.; Haddad, R.; Sarabia Neto, R. C.; Cosso, R. G.; Maia, D. R.; Maldaner, A. O.; Zacca, J. J.; Sanvido, G. B.; Romão, W.; Vaz, B. G.; Ifa, D. R.; Dill, A.; Cooks, R. G.; Eberlin, M. N.; Analyst 2010, 135, 2533 .

5. https://www.bcb.gov.br/novasnotas/assets/downloads/materialapoio/2e5/Cartilha.pdf, acessada em Março 2020.

6. Schmidt, E. M.; Franco, M. F.; Regino, K. G.; Lehmann, E. L.; Arruda, M. A. Z.; Rocha, W. F. C.; Fonsêca, R. B.; Souza Filho, W.; Eberlin, M. N.; Corrêa, D. N.; Sci. Justice 2014, 54, 459.

7. https://www.bcb.gov.br/content/estatisticas/mecirestfalsas/ EstatisticaFalsificacao_UFXDenominacao_internet_2018.pdf., acessada em Março 2020.

8. da Silva, V. A. G.; Talhavini, M.; Zacca, J. J.; Maldaner, A. O.; Peixoto, I. C. F.; Braga, J. W. B.; Microchem. J. 2014, 116, 235.

9. Correia, R. M.; Domingos, E.; Tosato, F.; Aquino, L. F. M.; Fontes, A. M.; Cáo, V. M.; Filgueiras, P. R.; Romão, W.; Forensic Chem. 2018, 8, 57.

10. Ulian, I. A.; Vidal, F. de B.; Resumo do XII Workshop de Visão Computacional, Campo Grande, Brasil, 2016.

11. Helfer, G. A.; Magnus, V. S.; Böck, F. C.; Teichmann, A.; Ferrão, M. F.; da Costa, A. B.; J. Braz. Chem. Soc. 2017, 28, 328.

12. Grasel, F. dos S.; de Oliveira, Tiago E.; Netz, P. A.; J. Braz. Chem. Soc. 2016, 27, 2372

13. Gonzalez, R. C.; Woods, R. E.; Processamento Digital de Imagens, $1^{\mathrm{a}}$ ed., Pearson Prentice Hall: São Paulo, 2007.

14. http://www.cbpf.br/cat/pdsi/pdf/ProcessamentoImagens.PDF, acessada em Março 2020.

15. Alves, E. T. S.; Dissertação de mestrado, Universidade Federal do Paraná, Brasil, 2011.

16. Bock, F. C.; Helfer, G. A.; da Costa, A. B.; Dessuy, M. B.; Ferrao M. F.; Food Anal. Methods 2018, 11, 1951.

17. Soares, S.; Lima, M. J.; Rocha, F. R.; Microchem. J. 2017, 133, 195.

18. Helfer, G. A.; Tischer, B.; Filoda, P. F.; Parckert, A. B.; Santos, R. B.; Vinciguerra, L. L.; Ferrão, M. F.; Barin, J. S.; Costa, A. B.; Food Anal. Methods 2018, 11, 2022.

19. Baumann, L.; Librelotto, M.; Pappis, C.; dos Santos, R. B.; Santos, R. O.; Helfer, G. A.; Lobo, E. A.; da Costa, A. B.; Águas subterrâneas 2019, 33, 1 . 